\title{
Food Suplements During Pregnancy Benefits and Risks: A Literature Review
}

\author{
Lili Stoilova, \\ Medical University of Plovdiv, Faculty of Pharmacy, Department of \\ Pharmacognosy and Pharmaceutical Chemistry, Plovdiv, Bulgaria \\ Boris Stoilov, \\ Medical University of Plovdiv, Faculty of Medicine, \\ Department of Obstetrics and Gynecology, Plovdiv, Bulgaria \\ Radiana Staynova, \\ Medical University of Plovdiv, Faculty of Pharmacy, \\ Department of Pharmaceutical Sciences, Plovdiv, Bulgaria
}

\begin{abstract}
Pregnancy is a physiological condition accompanied by a multitude of morphological, physiological and metabolic changes induced by the dynamic hormonal activity of the woman's body. These changes are associated with an increased need for nutrient intake. The lack of proper nutrition or irrational use of dietary supplements may have deleterious effects on both the mother and the developing fetus. The optimized intake of microelements and vitamins could be beneficial and even prevent common pathological conditions which frequently occur during pregnancy. The aim of the current review is to evaluate the potential benefits and risks associated with the intake of food supplements during pregnancy. Relevant publications were searched and analyzed using the following data bases: MEDLINE/PubMed, Scopus, Web of Knowledge, and Internet. Numerous authors support the opinion that the lack of obligatory quality control of food supplements is associated with many low-quality products which could have harmful health effects.
\end{abstract}

Keywords: Pregnancy, Nutrition, Micronutrients, Supplements, Risks

\section{Introduction}

Pregnancy is physiological condition associated with dynamic and anabolic processes and many physiologic adjustments that affect the metabolism of all nutrients. The main purpose of these adjustments is to support the fetal growth, the maternal homeostasis, and preparing the maternal organism for lactation (King 2000). 
- Morphological and physiological characteristics of the pelvic organs during pregnancy

The uterus is composed by three layers - endometrium, myometrium and serosa. The myometrium is mainly of smooth muscle tissue During pregnancy it undergoes hyperplasia and hypertrophy, greatly increasing its mass to accommodate the developing fetus. The cervix which connects the vaginal canal and the uterus is made up chiefly of collagen tissue. Unlike the myometrium, the cervix is much more denser and rigid which helps to support the expanding uterus. The lower segment of the uterus is formed by a junction called the isthmus. When cesarean section is performed, the incision is usually made through this particular area. The uterus is supplied with blood through the uterine arteries which branch out from the internal iliac arteries. They form a network in the uterine wall. Both sympathetic and parasympathetic innervation is present and it is often the subject of further studies as the finer functions of its motor pathways are yet to be fully clarified. It is known however that alpha-adrenergic receptor agonists stimulate the tissue of the myometrium whereas beta-adrenergic receptor agonists have inhibiting effect. Both pharmacological effects have been used in attempt to control premature labor. Through the pelvic splanchnic afferent fibers from the cervix enter the spinal cord at segments - S2,3,4. Nociception during labor is conveyed through the sympathetic plexus. The nerve fibers enter the spinal cord at the level of the thoracic segments (Magowan et al. 2009).

- The cervix: Its morphological and physiological characteristics change throughout the stages of pregnancy

In early pregnancy it is more vascular and less rigid. The secretions of the endocervical glands become more viscous and thicker. They serve as a barrier protecting the fetus from pathogens that may come from the vaginal canal. During the later stages of pregnancy, the cervix collagenous structure becomes much softer by absorbing polysaccharides (Magowan et al. 2009).

- Changes in bones and joints

These changes include the sacroiliac and symphyseal joints which become more extensible due to the action of the specific hormonal activity during pregnancy. This increased joint mobility facilitates labor but it may also be the cause for symphyseal pain.

- Changes in metabolism

There is an overall increased energy demand both for the fetus and the mother. This is followed by an increase of weight as the metabolic rate rises by an average of around 20 percent. Increased insulin sensitivity is also observed which in turn results in increased glycogen synthesis and accumulation of fat deposits and increased amino acid transfer in cells (Magowan et al. 2009). The overall morphological, physiological and metabolic changes which pregnant women undergo result in changes in blood 
pressure, fluid retention, anemia, constipation, cholestasis. These however are considered beneficial for the fetus (Magowan et al. 2009).

The mother's metabolism during pregnancy adjusts to meet the needs of the progressively growing fetus and sustain its development. Glucose and amino acid transfer from the mother to the fetus is most abundant while lipid transfer is present at a much lesser degree but it is not less essential than the other main nutrients (Hod et al. 2008). Fetal growth and development also depend on other essential nutrients, like vitamins. Many studies have shown that the deficiency of micronutrients such as magnesium, zinc and iron is associated with abnormal fetal development and risk of miscarriage ( Mensah et al. 2018 ). However, the importance of vitamin deficiency has been somewhat overlooked as well as the importance of the antioxidant status of the mother and the fetus. Studies have found that these factors are equally essential for the proper fetal development as the optimal intake of microelements. The concentration of vitamins in the mother's milk during breastfeeding is also dependent on their maternal serum levels. Other extra maternal factors include the consumption of constituents like phytanes and polyphenols which may inhibit the proper vitamin resorption. Even genetic polymorphism has been found to be a contributing factor (Hod et al. 2008). The importance of appropriate supplementation during pregnancy is undeniable, on the other hand recent studies have suggested that some food supplements could have a potential health risk (King 2003, Martello et al. 2007, Sanzini 2011).

\section{Materials and Methods}

Literature search was done using the following data bases: Medline/PubMed, Scopus, Web of Knowledge and Internet through the keywords "food supplements", "dietary supplements", "pregnancy", "nutrient intake", "maternal health", "nutrients", "fetal growth". The present systematic review is based on a total of 61 publications satisfying the search criteria. Our study was limited to the most commonly used food supplements by pregnant women.

\section{Discussion}

\section{Benefits of food supplements during pregnancy}

1. Folic acid

Folic acid (fig. 1) is (2S)-2-[[4-[[(2-Amino-4-oxo-1,4-dihydropteridin6-yl)methyl]amino] benzoyl]amino] pentanedioic acid. The substance is yellowish or orange, crystalline powder (European Pharmacopoeia 7.0 2016). Folic acid is an essential nutrient. It is important for DNA replication. It is a substrate for many enzymatic reactions involved in amino acid synthesis and vitamin metabolism (Greenberg et al. 2011). One-carbon transfer reactions, including purine and thymidylate biosynthesis, amino acid metabolism and 
formate oxidation are carried out with the presence of foliate (fig. 2). Purine and thymidylate biosynthesis are a fundamental requisite event underlying DNA and RNA synthesis (Hod et al. 2008). Demands for folate increase during pregnancy. It is important for growth and development of the fetus and for the maternal organism. The deficiency of folate is associated with abnormalities in maternal organism (anemia) and fetal development (Greenberg et al. 2011). The importance of the folic acid intake during/before pregnancy has been deeply discussed since 1960s (Hibbard 1964). The periconceptional intake of folic acid (400 $\mu \mathrm{g}$ daily) is associated with reduction in the risk of neural tube defects (Hibbard 1964; Shane 1995; Berry et al.1999). Figure 3 represents ultrasound picture of open spina bifida. In this condition, the spinal cord lies unprotected and exposed on the toxic effect of the amniotic fluid. These leads to damage of the spinal cord and the baby has problems with walking, passing urine and defecation. Open spina bifida is associated with central nerve system (CNS) abnormalities such as ventriculomegaly, hydrocephaly and "crush sign" at the back brain leading to additional damages. Additional intake should be at least one month prior to conception and should be continued throughout the first three months of pregnancy. Women who have had cases of previously affected children are recommended to take a higher dose of $4 \mathrm{mg}$ per day. Additional folate can be acquired through various sources such as foods rich in folate like greens broccoli, lettuce, etc. or by adding folic acid supplements to the diet. However, because dietary folate is less stable and has a lower bioavailability than supplementary folic acid, it is difficult to get enough folate from natural sources (Özek et al. 2008). Pregnant women who do not take extra folate supplements usually have decreased folate serum levels. This is due to a number of factors such as poor folate resorption, the increased folic needs of the fetus, increased catabolism, excretion and hormonal activity (Hod et al. 2008).

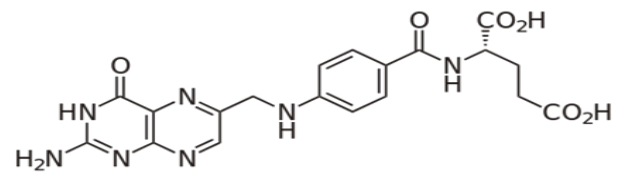

Figure.1 Structure of folic acid

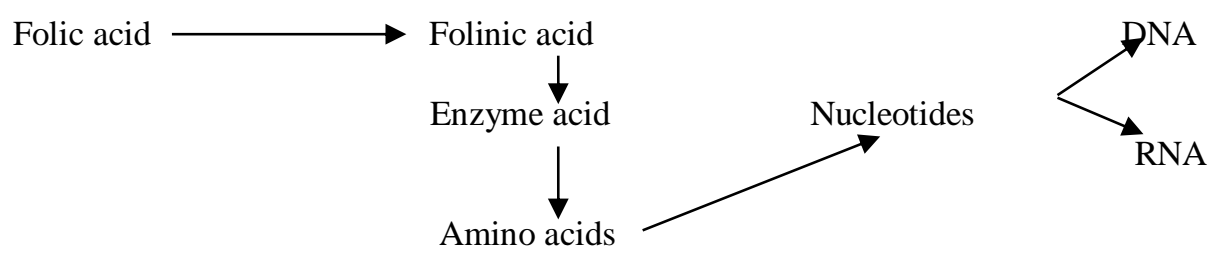

Figure 2. Importance of folic acid for DNA/RNA synthesis 
Folate is essential for rapidly proliferating tissues such as newly formed bone tissue as it is necessary for nucleic acid biosynthesis. With the introduction of folic acid supplements, folate deficiency nowadays is less common that it was in the past. The risk of developing anemia and neural tube defects during pregnancy is significantly lower. However, studies have found that women who are more prone to folate dietary deficiency are less likely to include folate supplements during the course of pregnancy. For that reason, folate is usually prescribed by doctors in combination with iron to compensate for such deficiencies (Hanretty 2004).

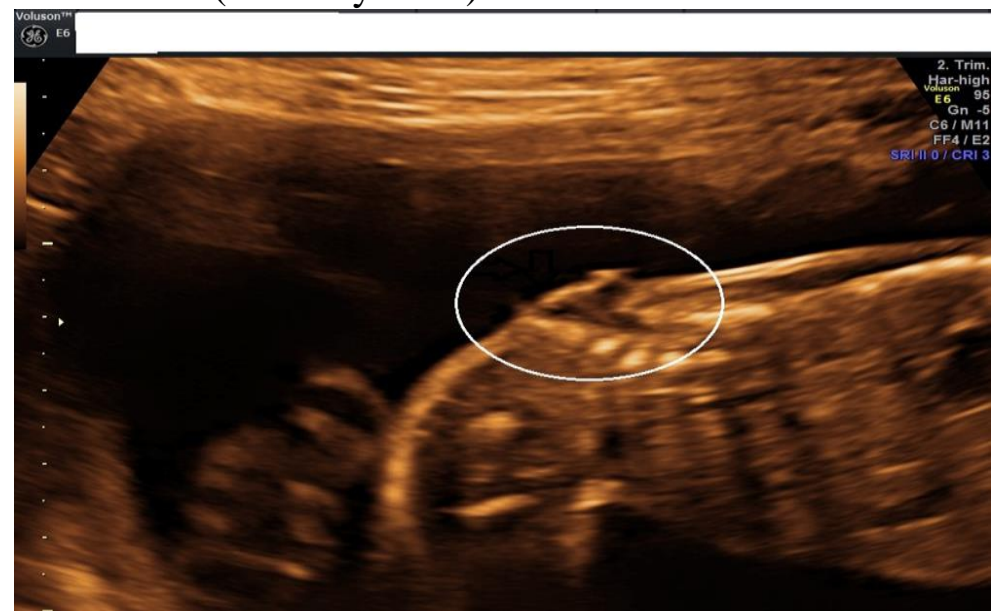

Figure 3. Ultrasound picture of open spina bifida - the surrounded area shows the defect of the spine and covering skin of the fetus.

\section{Micronutrients}

Micronutrients, known also as vitamins and minerals, have a significant impact on the health status of pregnant woman and the developing fetus (Black 2001). The physiological needs for micronutrients increase during the period of lactation as opposed to those before pregnancy. The need for calcium, phosphorus and magnesium rises by 40-50 percent as well as those of folic acid. The combination of prenatal multivitamin supplements with the addition of extra folic acid is recommended during lactation as well as increased fluid intake to compensate for dehydration during breastfeeding. Changes in the diet may also be necessary. Dietary deficiencies including total protein, amino acids and B vitamins may be observed especially in individuals who practice veganism. In such individuals appropriate corrections to the dietary regime should be made to include extra quantities of protein, iron, vitamins $B$ and D from vegan friendly sources like soya, nuts or other vegan combinations, while extra phytates and barn should be avoided (Gabbe et al. 2002). In some diets high in unrefined grains and legumes, the amount of nutrients consumed may be adequate, but dietary constituents, such as 
phytanes and polyphenols, can also limit their absorption. Here we summarize the main changes in the metabolism of the vitamins during pregnancy which are of major importance for the growth and development of the fetus (Hod et al. 2008). The deficiency of iron may have severely detrimental effects on both the fetus and the mother. Usually associated with anemia, iron deficiency, as well as folic acid deficiency, is also linked with an increased risk of hemorrhage during birth and congenital malformations in the fetus. Zinc deficiency may result in complications during pregnancy and delivery. Studies have found a connection between zinc deficiency and growth retardation, congenital abnormalities, delayed development of the immune and nervous systems of the fetus. Deficiency of iodine during pregnancy is associated with cretinism, possible fetal loss and premature birth. The shortage of micro elements such as magnesium, selenium, copper and calcium, is also associated with complications in pregnancy, birth or development of the fetus. Vitamin A or beta-carotene supplements use during pregnancy has been found to reduce maternal mortality by 50 per cent in one controlled study (Black 2001). Deficiencies of vitamins other than folate are associated with fetal pathology.

\section{Magnesium}

Magnesium is an essential microelement involved in many enzyme reactions (Makrides et al. 2014; Sanz 2011), acting as adenosine triphosphatase cofactor. It is crucial for energy-demanding metabolic processes as well as for protein synthesis and anaerobic phosphorylation. Magnesium homeostasis is maintained by the kidneys and small intestines. During conditions of magnesium shortage both organs increase the absorption of magnesium (Sanz 2011). Magnesium is involved in regulating body temperature, nucleic acid synthesis and protein metabolism. It also maintains the electrical potential of nerve and muscle cells. Pregnancy increases the need for magnesium however the majority of pregnant women rarely take action to compensate for those needs. Magnesium deficiency during pregnancy can be potentially detrimental to the health of both the mother and the fetus, with consequences that may affect the development of the child after birth (Lynne et al. 2016). Extra intake of magnesium during pregnancy shows significantly lower risk of maternal hospitalization, reduced risk of premature birth and lower need of intensive care for the newborn after birth (Spätling et al. 1988). Magnesium is necessary for the proper formation of bones and the various intracellular enzyme processes. Magnesium has established its role in obstetrics as it is an essential element for the development of the fetus. Magnesium deficiency may be associated with preeclampsia and premature birth and possibly low birth weight. Studies have found that magnesium deficiency during pregnancy significantly increases neonatal mortality and morbidity. Earlier studies conducted in developed countries have shown that 
proper magnesium intake during pregnancy significantly reduces the cases of hospitalization of pregnant women, premature labor, intrauterine growth retardation and frequent targeting of the newborn to the intensive care unit. In addition, the results show a significant impact on fetal and maternal morbidity both before and after birth. Positive correlation between magnesium intake in the first trimester of pregnancy with the birth weight is documented. However, magnesium supplements use during the second and third trimesters do not have any effect about the pregnancy outcome (Pathak et al. 2004). Recent studies show that the magnesium has neuroprotective effect on the infants when the pregnant woman was treated for preterm delivery or preeclampsia with magnesium sulfate. Additionally, the researchers found that the incidence of cerebral palsy for the same infants is reduced (Cunningham et al. 2014).

\section{Omega-3 fatty acids}

Omega-3 fatty acids are essential for the healty diet. Appropriate intake of omega-3 fatty acids is very important during pregnancy. They are fundamental components of the fetus' brain and retina. Omega-3 fatty acids may also play a role in determining the duration of pregnancy and in preventing perinatal depression. The most biologically active forms of omega3 fatty acids are docosahexaenoic acid (DHA) and eicosapentaenoic acid (EPA), which are derived predominantly from marine sources such as shellfish and algae (Coletta et al. 2010). Omega-3 is transferred from the mother to the fetus during pregnancy and from the mother to the infant after delivery through breastfeeding (Browne et al. 2006). The omega-3 fatty acids are essential nutrients and one of their important roles is the provision of 22 carbons and 6 double bonds known as DHA for the growth and function of nerve tissue. Insufficient intake of fatty acids-3 reduces DHA and increases omega- 6 fatty acids in the brain. The decrease in DHA in the developing brain leads to deficiencies in neurogenesis, the metabolism of neurotransmitters, and altered visual function in animals. Western diets are low in omega-3 fatty acids, including the 18 carbons carbon-3 alpha-linolenic acids, mainly found in vegetable oils, and DHA, which is mostly found in fish. The state of DHA in the newborn and the infant depends on the maternal intake of DHA and varies widely. Epidemiological studies associate low DHA to mother with an increased risk of poor development of the nervous system. Intervention studies have shown that improving maternal nutrition with DHA reduces the risk of visual and neural development defects of the neonate and infant. There is sufficient evidence to conclude that the maternal diet rich of fatty acids is important for the transfer of DHA to the baby before and after birth, with short and long-term effects on neuronal function. However, genetic variations in the genes encoding fatty acid desaturases also influence the essential metabolism of fatty acids and may increase the requirements in some individuals. It is 
important to include omega-3 fatty acids, for brain development of the fetus and the optimization of omega- 3 and omega- 6 fatty acids during pregnancy and lactation, as well as the fatty acids nutrients for intravenous and formulafeed neonates (Innis 2008). The placenta regulates the nutritional, immunological and endocrine system of the fetus. The importance of fatty acids known to have immunomodulatory effects through the production of eicosanoids may end during pregnancy. Its complex metabolism varies from the deposition in maternal tissues at the beginning of pregnancy to the release during the last stage of pregnancy, to meet the fetal requirements. Previous attempts to modify the feeding of the pregnant woman and, therefore, the nutritional and immunological environment of the fetus are focused on the addition of long-chain polyunsaturated fatty acids (PUFA), fish oil and probiotic bacteria. Its synergistic effects suggest the need to look at the general diet (Kaplas et al. 2007). Depression during pregnancy affects both the mother and the child. The omega-3 PUFA with a possible antidepressant effect and lack of teratogenicity for the fetus, seem to be a beneficial alternative for the treatment (Chiu et al. 2003).

\section{Probiotics}

The gastrointestinal tract acts as a barrier against potentially harmful antigens from microorganisms that may come from the food. The adequate physiological immune response in the gastrointestinal tract depends on the localization and the condition of the intestinal microflora. A new therapeutic approach in this area has been applied and it relies on the introduction of beneficial micro flora to the body designated as probiotics. A possible mechanism of probiotic therapy is the promotion of a non-immunological intestinal protective barrier, which includes normalizing the increased intestinal permeability and altered intestinal microecology. Another possible mechanism of probiotic therapy is to improve the immunological barrier of the intestine, in particular by intestinal immunoglobulin A responses and to alleviate intestinal inflammatory reactions that produce intestinal stabilizing effects. Many probiotic effects are mediated by immune regulation, in particular by controlling the balance of proinflammatory and antiinflammatory cytokines (Isolauri et al. 2001; Lukova et al. 2018). Interaction between hereditary factors and environmental factors appears to affect every stage of development from conception to the early postnatal period with potential long-term effects on the health of children and adults. During pregnancy, the immune and metabolic functions of the fetus depend on the mother. In addition, improving these functions seems to start in the uterus and is sensitive to the diet. Microbial life in the intestinal tract develops a number of physiological roles in the human body that affect both metabolic and immune functions, especially in early neonatal life and possibly even in the 
womb. The transmission of bacteria from the mother to the newborn by direct contact with the mother's microbe at birth and breastfeeding during lactation period also appears to affect colon colonization with potential health consequences (Sanz 2011). Complications of pregnancy are associated with adverse outcomes for mother and baby in the short and long term. The probiotic supplements can help regulate the unbalanced microflora composition observed in obesity and diabetes (Arango et al. 2015). The prevalence of atopic diseases is increasing throughout the Western world, and primary prevention is needed to reverse this trend. The role of breastfeeding, the best source of nutrition for babies, in the protection against atopic disease remains elusive. The use of probiotics in pregnant and nursing women has been shown to increase the immunoprotective potential of breast milk as assessed by the amount of anti-inflammatory $\beta 2$ (TGF- $\beta 2$ ) growth factor in milk (Rautava et al. 2002). Mammary glands can be affected by several factors, such as birth, breastfeeding, mother's lifestyle, health status, and diet. Numerous studies have shown a variety of positive effects from the use of probiotics during pregnancy and breastfeeding for the mother and the newborn (Treven et al. 2015). Lactic acid bacteria and bifidobacteria are increasingly being applied to pregnant women and infants with the intention of improving health. Although these organisms have a long record for safe use, it is important to identify all adverse effects in potentially vulnerable populations (Allen et al. 2010). Supplementation with probiotics during pregnancy has the potential to affect the immune parameters of the fetus as well as immunomodulatory factors in breast milk (Prescott et al. 2008).

\section{Others}

\subsection{Iodine supplementation}

Maternal iodine is necessary for the normal development of the child's brain during fetal and early postnatal life. For that reason, maternal iodine requirements increase during pregnancy and breastfeeding. Iodine requirements are 250-300 g/day during pregnancy, 225-350 g/day during breastfeeding, and $90 \mathrm{~g} / \mathrm{day}$ during the neonatal period. It is observed that there is increased requirement for maternal iodine due to the transfer of thyroxine (T4) and iodide from mother to fetus during pregnancy and loss of iodide in breast milk during breastfeeding. These however are necessary to ensure normal brain development and to prevent mental retardation in the child. The requirement for iodine increases during pregnancy due to (Delange 2004):

- $\quad$ increased requirement for T4 to maintain normal generalmetabolism in the mother;

- $\quad$ the transfer of T4 and iodide from the mother to the fetus;

- $\quad$ increased iodide loss through the kidneys due to increased renal clearance of iodide; 
The main change in thyroid function associated with pregnancy is the requirement for increased thyroid hormone production, which depends directly on the adequate availability of dietary iodine and the integrity of the glandular mechanism. Physiological adaptation occurs when iodine intake is adequate, and this is replaced by pathological changes when iodine deficiency occurs. Consequently, pregnancy typically acts as a revealer of major iodine restriction. Iodine deficiency (ID) has important consequences for both the mother and the fetus, resulting in permanent glandular stimulation, hypothyroxinemia and goitrogenesis. Furthermore, since severe identification may be associated with impairment of the psycho-neuro-intellectual outcome in offspring as the mother and offspring are exposed to ID during pregnancy (and postnatal period). Since ID still prevails today in several European countries - it is proposed to add iodine supplements to pregnant and nursing women in the early 1990s (Glinoer 2004). The fetus is completely dependent at the beginning of pregnancy from maternal thyroxine for normal brain development. Adequate maternal iodine delivery during pregnancy is essential for the production of thyroxine in the mother and later for thyroid function in the fetus. If iodine failure results in insufficient thyroid hormone production and hypothyroidism during pregnancy, irreversible damage to the fetal brain may occur. The American Thyroid Association recommends that women receive $150 \mu \mathrm{g}$ iodine supplements daily during pregnancy and lactation and that all prenatal vitamin-mineral preparations contain $150 \mu \mathrm{g}$ of iodine (Becker et al. 2006).

\subsection{Calcium supplementation}

Calcium homeostasis is maintained by the parathyroid hormone (PTH) and 1,25-dihydroxyvitamin $\mathrm{D},(1,25[\mathrm{OH}] 2 \mathrm{D} 3)$. About 50 percent of serum calcium is bound to proteins (mostly albumin), 10 percent in the form of anionic complexes, and $40 \%$ circulates freely as ionized calcium. During pregnancy there is an active transfer of maternal calcium to the fetus. Before birth, the fetus needs 25 to $30 \mathrm{~g}$ of calcium for bone mineralization. Total serum calcium during pregnancy is 8 per cent below the level after delivery . The upper limit of normal is $9.5 \mathrm{mg}$ per milliliter. This reduction in total serum calcium is due to physiological hypoalbuminemia, which is secondary to the normal widening of the intravascular volume observed at the beginning of pregnancy. However, the ionized calcium levels remain unchanged during pregnancy. Serum levels of calcitonin are higher during pregnancy and postpartum than in non-pregnancy controls. In 20 per cent of patients, the values exceed the normal range of pregnancy (Gabbe et al. 2002). Calcium metabolism during pregnancy is a complex process involving calcium, phosphorus, vitamin D, parathyroid hormone (PTH) and calcitonin $(\mathrm{CT})$. The absorption of calcium is increased during pregnancy and probably increased 
storage in the maternal skeleton. Adequate amounts are provided by the current recommended dietary allowance of $1200 \mathrm{mg}$ daily, which can easily be satisfied with natural foods, especially milk. If additional calcium is given, it is probably advisable to use nonphosphate salt. Some evidence suggests that excessive phosphate intake may be associated with leg cramps during pregnancy (Pitkin 1975). Preeclampsia and eclampsia are common causes of serious morbidity and death. Calcium supplementation can reduce the risk of preeclampsia and can help prevent premature birth. Calcium supplementation ( $\geq 1 \mathrm{~g} /$ day) is associated with a significant reduction in the risk of preeclampsia, especially in women with low calcium levels. It also reduces premature birth and the occurrence of a combined outcome of "maternal death or serious morbidity". The World Health Organization recommends calcium $1.5 \mathrm{~g}$ to $2 \mathrm{~g}$ per day for pregnant women with low dietary calcium intake (Hofmeyr et al. 2014). During pregnancy there are considerable changes in maternal calcium metabolism and serum levels of vitamin D due to the fetal bone mineral accretion. During the last trimester the fetus receives roughly 25$30 \mathrm{~g}$ of calcium. Additional quantities of the microelement may be acquired through increased intestinal absorption from calcium rich foods, increased renal conservation and increased bone mobilization (Hod et al. 2008).

\subsection{Vitamin D supplementation}

The initial studies on the importance of vitamin $\mathrm{D}$ have been limited to its role in regulating calcium and phosphate levels, and its involvement in the pathogenesis of rickets. Further studies however have found the presence of vitamin D receptors linked to chromosomes in almost every cell and tissue in the body, thus leading to its important effects on different organs. These effects and the fact that vitamin D is made in the skin by ultraviolet-B (UVB) radiation followed by a thermal process, and that the active vitamin $D$ circulates in the blood created a new understanding of vitamin D as a hormone. Vitamin D is crucial during pregnancy as the mother is the sole source of vitamin D substrate for the developing fetus. While vitamin D status during pregnancy varies worldwide as it depends on the degree of sunlight exposure, degree of skin pigmentation, latitude, lifestyle, body mass index and the intake of oral vitamin D supplements, it is clear that women with darker pigmentation and limited sunlight exposure are at greatest risk for vitamin D deficiency. It is established that if a woman is deficient during her pregnancy, her fetus also will be deficient during gestation (Delange 2004). Vitamin D is known to be involved in skeletal homeostasis during pregnancy, and severe vitamin D deficiency may lead to neonatal seizures in neonates with profound hypocalcemia (Hollis et al. 2011). During pregnancy and lactation, the mother requires significant amounts of calcium to pass on to the developing fetus and suckling neonate, respectively. Given that adult calcium and bone metabolism 
depend on vitamin D sufficiency, it would seem to be especially critical during pregnancy and lactation. The vitamin D-deficient or insufficient neonate is at risk of problems, including hypocalcemia and rickets. Due to poor penetrance of vitamin D and 25-hydroxyvitamin D [25(OH)D] into milk, exclusively breastfed infants are at higher risk of vitamin $\mathrm{D}$ deficiency than are formula-fed infants (Kovacs 2008). The main function of vitamin D is to maintain calcium and phosphate homeostasis so that their serum levels remain optimal for normal neuromuscular function and bone mineralization. Other than that, however it has been established that vitamin D metabolites have additional physiological and pharmacological effects. Vitamin D undergoes biosynthesis from 7-dehydrocholesterol in the epidermis under the influence of ultraviolet radiation but it can also be acquired through proper diet. It is subsequently transported to the liver where it undergoes hydroxylation which results in its inactive form 25-hydroxyvitamin $D$. In the kidneys the active form of vitamin D - 1,25-dihydroxyvitamin D is formed through further hydroxylation. The active form increases intestinal calcium absorption, decreases renal excretion and under the influence of the parathyroid hormone activates calcium bone mobilization. Significant changes in maternal vitamin $\mathrm{D}$ and calcium metabolism occur during pregnancy to provide the calcium needed for fetal bone mineral accretion (Hod et al. 2008).

\section{Food supplements use during pregnancy: risks}

Food supplements represent concentrated sources of nutrients like minerals, vitamins, amino acids, omega fatty acids and/or other substances (EFSA, Crişan, 2012). These products are intended only to enrich the diet. They are not medicinal products and should not pretend for health claims (EFSA). The food supplement market has continuously grown in the last ten years not only in the European Union but also all over the World (Crişan 2012; Strano-Rossi et al. 2015). In the European Union, food supplements are regulated as foods (EFSA). The current legislation of the European Union is designed to protect consumers from misleading and false claims (Nocella and Kennedy 2012) but at the same time there is not obligatory analytical control (Ivanova 2017). The lack of obligatory quality control has led to many cases of undeclared substances in food supplements (Neves and Caldas 2017). Recent studies have declared results that dietary supplements might contain undeclared substances in levels that could represent potential health risks (Venugopal et al. 1996; Ernst 2002; Martello et al. 2007; Ancuceanu et al. 2013; Haneef et al. 2013; Venhuis et al. 2014; Gaudiano et al. 2016; da Justa et al 2017; Petkova-Gueorguieva et al. 2018). Data from modern studies show that customers believe that food supplements are safe products and even safer than conventional drugs (Petkova - Gueorguieva et al. 2018). In the last decade, many patients believe that conventional drugs should be avoided 
because they "might" cause autism (vaccines) (Peteva et al. 2016), toxic effects or some serious side effects. The conventional drugs are essential for the quality of humans' life. These products have prolonged and imploved the quality of humans' life. The medicinal products are used for the treatment of different pathologies and they have predictable side effects. The quality control for medicinal products is obligatory all over the world and it is applied throughout all processing stages - from the drug substance to the finished product. Many analytical techniques like High-performance liquid chromatography (HPLC) and Gas chromatography are involved in the quality control of drugs. There is also an obligatory post-marketing control. Most customers consider that food supplements are pharmaceutical products with high quality ( Petkova - Gueorguieva et al. 2018). The analytical control of food supplements is essential for customer safety. It is very important the quality control to be obligatory and to be applied throughout all processing stages - from the raw material to the finished product (Petkova-Gueorguieva et al 2018; Sanzini 2011). Unfortunately, the analytical control for these products is not obligatory (Ivanova 2017). Most of the food supplements are either poorly monitored or not even monitored at all (Ekor 2014). The adequate supply of nutrients is probably one of the most important factors affecting pregnancy outcome (Kamber et al. 2001; Halsted 2003). Poor intakes of iron, folic acid, magnesium, calcium and other nutrients induce a poor maternal status and could be associated with some serious problems (Ekor 2014, Martínez-Sanz et al. 2017). It is important for the pregnant woman to have an adequate diet but also it is important the diet to be enriched with food supplements. It is important the food supplements to be provided by reliable manufacturers, who have a GMP certificate, traditions in the manufacturing and quality control systems.

\section{Conclusion}

Pregnancy is a physiological condition, associated with dynamic processes and many physiologic adjustments that affect the metabolism of all nutrients. The main purpose of these adjustments is to support the fetal growth and the maternal homeostasis. Poor intake of some nutrients is associated with serious health consequences during pregnancy. The diet of pregnant woman is important to be enriched with food supplements containing some important nutrients for both the maternal and fetal organism. It is important to note that food supplements are not pharmaceutical products. The quality of the food supplements is not supposed to be the same as that of the conventional drugs. In the European Union and in the most part of the World these products are regulated as "foods". There is not obligatory analytical control (quality and quantity) for food supplements. This is associated with many cases of poor quality of food supplements and that could cause some negative health 
consequences. It would be very dangerous for a pregnant woman to intake products with poor quality. It is important the food supplements to be provided by reliable manufacturers, who have a GMP certificate, traditions in the manufacturing and reliable quality control systems.

\section{References:}

1. Sutton, R. A. (1994). Magnesium deficiency: pathophysiologic and clinical overview. American Journal of Kidney Diseases, 24(5), 737752.

2. Allen, S. J., Jordan, S., Storey, M., Thornton, C. A., Gravenor, M., Garaiova, I., ... \& Morgan, G. (2010). Dietary supplementation with lactobacilli and bifidobacteria is well tolerated and not associated with adverse events during late pregnancy and early infancy. The Journal of nutrition, 140(3), 483-488.

3. Ancuceanu, R., Dinu, M., \& Arama, C. (2013). Weight loss food supplements: adulteration and multiple quality issues in two products of Chinese origin. Farmacia, 61(1), 28-44.

4. Arango, L. F. G., Barrett, H. L., Callaway, L. K., \& Nitert, M. D. (2015). Probiotics and pregnancy. Current diabetes reports, 15(1), 567.

5. Baylis, A., Cameron-Smith, D., \& Burke, L. M. (2001). Inadvertent doping through supplement use by athletes: assessment and management of the risk in Australia. International journal of sport nutrition and exercise metabolism, 11(3), 365-383.

6. Becker, D. V., Braverman, L. E., Delange, F., Dunn, J. T., Franklyn, J. A., Hollowell, J. G., ... \& Rovet, J. F. (2006). Iodine supplementation for pregnancy and lactation-United States and Canada: recommendations of the American Thyroid Association. Thyroid, 16(10), 949-951.

7. Berry, R. J., et al. (1999). Prevention of neural-tube defects with folic acid in China. New England journal of medicine, 1485-1490.

8. Black, R.E. (2001). Micronutrients in pregnancy. British Journal of Nutrition, 85.S2: S193-S197.

9. Browne, J. C., Scott, K. M., \& Silvers, K. M. (2006). Fish consumption in pregnancy and omega-3 status after birth are not associated with postnatal depression. Journal of affective disorders, 90(2-3), 131-139.

10. Buell, J. L., Franks, R., Ransone, J., Powers, M. E., Laquale, K. M., \& Carlson-Phillips, A. (2013). National Athletic Trainers' Association position statement: evaluation of dietary supplements for performance nutrition. Journal of athletic training, 48(1), 124-136

11. Chiu, Chih-Chiang, et al. (2003). Omega-3 fatty acids for depression in pregnancy." American journal of psychiatry, 160(2): 385-385. 
12. Cohen, P. A., Travis, J. C., Keizers, P. H., Boyer, F. E., \& Venhuis, B. J. (2019). The stimulant higenamine in weight loss and sports supplements. Clinical toxicology, 57(2), 125-130.

13. Coletta, J. M., Bell, S. J., \& Roman, A. S. (2010). Omega-3 Fatty acids and pregnancy. Reviews in obstetrics \& gynecology, 3(4), 163-171.

14. Crişan, O. F. E. L. I. A. (2012). Health claims in food supplement advertising. Farmacia, 60(1), 138-142.

15. Cunningham F. et al. (2014) Williams Obstetrics 24th ed., McGrawHill Education, 779-800

16. Dalton, L. M., Ní Fhloinn, D. M., Gaydadzhieva, G. T., Mazurkiewicz, O. M., Leeson, H., \& Wright, C. P. (2016). Magnesium in pregnancy. Nutrition reviews, 74(9), 549-557.

17. Delange, F. (2004). Optimal iodine nutrition during pregnancy, lactation and the neonatal period. Int J Endocrinol Metab, 2(1), 1-12.

18. Ekor, M. (2014). The growing use of herbal medicines: issues relating to adverse reactions and challenges in monitoring safety. Frontiers in pharmacology, 4, 177.

19. Ernst, E. (2002). Toxic heavy metals and undeclared drugs in Asian herbal medicines. Trends in pharmacological sciences, 23(3), 136139.

20. Europarat [European Department for the Quality of Medicines] (2016) European Pharmacopoeia 7.0. Council of Europe, Strasbourg.

21. Food supplements .https://www.efsa.europa.eu/en/topics/topic/foodsupplements

22. Gabbe et al.(2002) Obstetrics - Normal and Problem Pregnancies, 4th ed., Copyright Churchill Livingstone, Inc, 131-132, 1004-1005

23. Gaudiano, M. C., Manna, L., Bartolomei, M., Rodomonte, A. L., Bertocchi, P., Antoniella, E., ... \& Valvo, L. (2016). Health risks related to illegal and on-line sale of drugs and food supplements: results of a survey on marketed products in Italy from 2011 to 2013. Annali dell'Istituto superiore di sanita, 52(1), 128-132.

24. Glinoer, D. (2004). The regulation of thyroid function during normal pregnancy: importance of the iodine nutrition status. Best practice \& research Clinical endocrinology \& metabolism, 18(2), 133-152.

25. Greenberg, J. A., Bell, S. J., Guan, Y., \& Yu, Y. H. (2011). Folic acid supplementation and pregnancy: more than just neural tube defect prevention. Reviews in Obstetrics and Gynecology, 4(2), 52.

26. Gurley, BJ., Gardner SF, Hubbard MA. (2000). Content versus label claims in ephedra-containing dietary supplements. American Journal of Health-System Pharmacy 57;(57): 963-969. 
27. Halsted, CH. (2003). Dietary supplements and functional foods: 2 sides of a coin? The American journal of clinical nutrition 77(4 Suppl): 1001S-1007S

28. Haneef, J. , Shaharyar, M. , Husain, A. , Rashid, M. , Mishra, R. , Siddique, N. A. and Pal, M. (2013), Analytical methods for the detection of undeclared synthetic drugs in traditional herbal medicines as adulterants. Drug Test. Analysis, 5: 607-613. doi:10.1002/dta.1482

29. Hanretty KP. (2004). OBSTETRICS ILLUSTRATED Sixth edition, Elsevier Limited. 140-141

30. Hibbard, B. M. (1964), THE ROLE OF FOLIC ACID IN PREGNANCY*. BJOG: An International Journal of Obstetrics \& Gynaecology, 71: 529-542. doi:10.1111/j.1471-0528.1964.tb04317.x

31. Hod M. et al.(2008). Diabetes and Pregnancy, Second Edition, Informa UK Ltd, 29-48

32. Hofmeyr, G.J, Lawrie TA, Atallah AN, Duley L, Torloni MR. (2014) Calcium supplementation during pregnancy for preventing hypertensive disorders and related problems. Cochrane database of systematic reviews. 24;(6):CD001059. doi: 10.1002/14651858.CD001059.pub4

33. Hollis, B. W., Johnson, D., Hulsey, T. C., Ebeling, M., \& Wagner, C. L. (2011). Vitamin D supplementation during pregnancy: doubleblind, randomized clinical trial of safety and effectiveness. Journal of bone and mineral research : the official journal of the American Society for Bone and Mineral Research, 26(10), 2341-2357. doi:10.1002/jbmr.463

34. Innis, S. M. (2008). Dietary omega 3 fatty acids and the developing brain. Brain $\quad$ research.27;1237 $43 . \quad$ doi:

10.1016/j.brainres.2008.08.078

35. Isolauri, E., Sütas Y, Kankaanpää P, Arvilommi H, Salminen S. (2001) Probiotics: effects on immunity.The American journal of clinical nutrition 73 (2 Suppl): 444s-450s. doi: 10.1093/ajcn/73.2.444s.

36. Ivanova S, Ivanov K, Gueorgiev S, Petkova E. (2017). UHPLC/MS detection of yohimbine in food supplements. Biomedical Research, 28(7):3281-3285.

37. Kamber M Baume N, Saugy M, Rivier L. (2011). Nutritional supplements as a source for positive doping cases?." International journal of sport nutrition and exercise metabolism, 11(2): 258-263.

38. Kaplas, N., Isolauri, E., Lampi, AM. et al. (2007). Dietary counseling and probiotic supplementation during pregnancy modify placental phospholipid fatty acids. Lipids. 42(9): 865-870. 
39. King, J. C. (2000). Physiology of pregnancy and nutrient metabolism. The American journal of clinical nutrition, 71(5):1218S-1225S. https://doi.org/10.1093/ajcn/71.5.1218s

40. Kovacs, C. S. (2008). Vitamin D in pregnancy and lactation: maternal, fetal, and neonatal outcomes from human and animal studies. The American journal of clinical nutrition, 88(2), 520S-528S.

41. Magowan, B. A., Owen, P., \& Thomson, A. (2014). Clinical obstetrics and gynaecology E-Book. Elsevier Health Sciences.

42. Makrides, M., Crosby, D. D., Bain, E., \& Crowther, C. A. (2014). Magnesium supplementation in pregnancy. Cochrane Database of Systematic Reviews, (4).

43. Martello, S., Felli, M., \& Chiarotti, M. (2007). Survey of nutritional supplements for selected illegal anabolic steroids and ephedrine using LC-MS/MS and GC-MS methods, respectively. Food additives and contaminants, 24(3), 258-265.

44. Martínez-Sanz, J., Sospedra, I., Ortiz, C., Baladía, E., Gil-Izquierdo, A., \& Ortiz-Moncada, R. (2017). Intended or unintended doping? A review of the presence of doping substances in dietary supplements used in sports. Nutrients, 9(10), 1093.

45. Mensah, T. R., Eklou-Lawson, M., Gbeasor-Komlanvi, F. A., Ekouevi, D. K., Karou, D. S., Ameyapoh, Y., \& de Souza, C. A. (2018). Assessment of Knowledge and Consumption Patterns of Soybean, Moringa Oleifera, Aloe Vera and Other Agro-Resources: The Case of the Elderly and Pregnant/Nursing Women in Lome, Togo. European Scientific Journal, ESJ, 14.

46. Neves DBDJ, Caldas ED. (2017). Determination of caffeine and identification of undeclared substances in dietary supplements and caffeine dietary exposure assessment. Food and Chemical Toxicology. 105: 194-202. doi: 10.1016/j.fct.2017.03.063

47. Nocella, G., \& Kennedy, O. (2012). Food health claims-what consumers understand. Food Policy, 37(5), 571-580.

48. Özek, M. M., Cinalli, G., Maixner, W. J., \& Maixner, W. (Eds.). (2008). Spina bifida: management and outcome. Springer Science \& Business Media.

49. Lukova, P., Karcheva-Bahchevanska, D., Mollova, D., Nikolova, M., Mladenov, R., \& Iliev, I. (2018). Study of prebiotic potential and antioxidant activity in Plantago spp. leaves after enzymatic hydrolysis with hemicellulase and xylanase. Engineering in Life Sciences, 18(11), 831-839.

50. Pathak, P., \& Kapil, U. (2004). Role of trace elements zinc, copper and magnesium during pregnancy and its outcome. The Indian Journal of Pediatrics, 71(11), 1003-1005. 
51. Peteva, G., Kevorkian, A., Boikinova, O., Baltadjiev, I., Ivanova, S., Ivanov K. (2016). Reasons to vaccinate. Pediatriya, 56 (1), 32-38

52. Petkova-Gueorguieva, E., Ivanov, K., Gueorguiev, S., Mihaylova, A., Madzharov, V., \& Ivanova, S. (2018). Detection of sibutramine in herbal food supplements by UHPLC/HRMS and UHPLC/MSMS. Biomedical Research, 29(14), 3006-3009.

53. Petroczi, A., Taylor, G., \& Naughton, D. P. (2011). Mission impossible? Regulatory and enforcement issues to ensure safety of dietary supplements. Food and Chemical Toxicology, 49(2), 393-402.

54. Pitkin, R. M. (1975). Calcium metabolism in pregnancy: a review. American journal of obstetrics and gynecology, 121(5), 724737.

55. Prescott, S. L., Wickens, K., Westcott, L., Jung, W., Currie, H., Black, P. N., .. \& Wu, L. (2008). Supplementation with Lactobacillus rhamnosus or Bifidobacterium lactis probiotics in pregnancy increases cord blood interferon- $\gamma$ and breast milk transforming growth factor- $\beta$ and immunoglobin $A$ detection. Clinical \& Experimental Allergy, 38(10), 1606-1614.

56. Rautava, S., Kalliomäki, M., \& Isolauri, E. (2002). Probiotics during pregnancy and breast-feeding might confer immunomodulatory protection against atopic disease in the infant. Journal of allergy and clinical immunology, 109(1), 119-121.

57. Sanz, Y. (2011). Gut microbiota and probiotics in maternal and infant health. The American journal of clinical nutrition, 94(suppl_6), 2000S-2005S.

58. Sanzini, E., Badea, M., Dos Santos, A., Restani, P., \& Sievers, H. (2011). Quality control of plant food supplements. Food \& function, 2(12), 740-746.

59. Shane, B. (1995). Folate chemistry and metabolism. Folate in health and disease, 1-22.

60. SPÄTLING， L.， \& SPÄTLING， G. (1988). Magnesium supplementation in pregnancy. A double-blind study. BJOG: An International Journal of Obstetrics \& Gynaecology, 95(2), 120-125.

61. Strano-Rossi, S., Odoardi, S., Castrignanò, E., Serpelloni, G., \& Chiarotti, M. (2015). Liquid chromatography-high resolution mass spectrometry (LC-HRMS) determination of stimulants, anorectic drugs and phosphodiesterase 5 inhibitors (PDE5I) in food supplements. Journal of pharmaceutical and biomedical analysis, 106, 144-152.

62. Treven, P., Mrak, V., Matijašić, B. B., Horvat, S., \& Rogelj, I. (2015). Administration of probiotics Lactobacillus rhamnosus GG and Lactobacillus gasseri K7 during pregnancy and lactation changes 
mouse mesenteric lymph nodes and mammary gland microbiota. Journal of dairy science, 98(4), 2114-2128.

63. Venhuis, B., Keizers, P., van Riel, A., \& de Kaste, D. (2014). A cocktail of synthetic stimulants found in a dietary supplement associated with serious adverse events. Drug testing and analysis, 6(6), 578-581.

64. Venugopal, R., Tollefson, L., Hyman, F. N., Timbo, B., Joyce, R. E., \& Klontz, K. C. (1996). Recalls of foods and cosmetics by the US Food and Drug Administration. Journal of food protection, 59(8), 876-880. 